\title{
Experimental Evidence of a Double Layer in a Large Volume Helicon Reactor
}

\author{
O. Sutherland, ${ }^{1}$ C. Charles, ${ }^{1}$ N. Plihon, ${ }^{1,2}$ and R. W. Boswell ${ }^{1}$ \\ ${ }^{1}$ Space Plasma and Plasma Processing Group, Plasma Research Laboratory, Research School of Physical Sciences and Engineering, \\ Australian National University, Acton 0200, Canberra, Australia \\ ${ }^{2}$ Laboratoire de Physique et Technologie des Plasmas, Ecole Polytechnique, 91128 Palaiseau, Paris, France
}

(Received 4 April 2005; published 7 November 2005)

\begin{abstract}
The self-consistently generated current-free electric double layer (DL) is shown to scale up with the source tube diameter and appears not to be affected by $\mathrm{rf}$ driving frequency and changes in reactor geometry. This Letter presents the first simultaneous measurements of local plasma potential and beam energy as a function of axial position. The DL is shown to be no more than $5 \mathrm{~mm}$ thick (20 D lengths) and positioned just downstream of the maximum in the magnetic field gradient. Furthermore, its position relative to the magnetic field is observed to be invariant as the magnetic field is translated axially. Measurements of the potential drop across the DL are presented for pressures down to 0.09 mTorr and the DL strength $\left(\phi_{\mathrm{DL}} / T_{e}\right)$ is determined to be between 5 and 7 .
\end{abstract}

PACS numbers: 52.50.Qt

Electric double layers (DLs) are observed in nearly collisionless plasmas and are spatially limited changes in potential, which often separate two different types of plasma. They are important as energy dissipation mechanisms and have been associated with the acceleration of charged particles along magnetic fields. Several authors have invoked DLs to explain astrophysical phenomena including reconnection events, solar flares, and aurora [1,2], as these events require the rapid change of electromagnetic energy into particle energy. Numerous satellite based experiments have revealed the existence of strong "shocklike" variations in potential and corresponding beams of high energy particles in the magnetosphere [3-5].

More recently, the electric field embedded in the DL has been proposed as a thrust producing mechanism for space propulsion especially in light of the relative simplicity and anticipated reliability of the underlying technology [6]. Large diameter beams of low energy ions are also of considerable interest to the surface treatment and metrology community for large-scale processing of surfaces.

Since in situ measurements are difficult and costly in space, much of the current knowledge on DLs has been guided by laboratory experiments [7-10], theoretical work [8,11], and computer simulation [12-14]. Historically, experimental regimes have fallen into two main categories, namely, double or triple plasma devices, which are voltage driven $[15,16]$, and current driven discharges with an abrupt change in diameter [17], where the common thread was the requirement of an artificially induced current to drive the DL. However, by carefully adjusting the potentials on immersed grids, it has been shown that it is the flow of ions and electrons rather than the applied potentials in triple plasma devices which create the DL [18]. Previous research on the free expansion of plasmas has also demonstrated the existence of a current-free double layer [19] and explained its physics based on a two-electron population. DLs have also been measured in electronegative plasmas in the absence of a magnetic field [20]. More recently, interest has turned towards the effect of inhomogenous magnetic fields on the dynamics and stability of DLs $[21,22]$. In these experiments, the DL is created independently (by some form of current drive) and the magnetic field is used to control its position. In 2003, it was demonstrated that a current-free double layer could be created self-consistently using a helicon source and an expanding magnetic field [6]. This work was significant because it presented the first laboratory demonstration a selfgenerating current-free stationary electric DL and showed the important role of an expanding magnetic field in the self-consistent formation of the structure. Subsequent studies akin to this one confirmed the presence of an ion beam under similar experimental conditions [23,24] using laser induced fluorescence; however, Sun et al., in particular, reported that it was the neutral pressure more than the magnetic field which had the most influence on the energy of the beam ions and that the DL thickness might be as high as $500 \mathrm{D}$ lengths [23]. This was in stark contrast to Ref. [6], in which, below a certain threshold neutral pressure, the magnetic field was the most sensitive parameter and where the DL thickness was reported to be on the order of $50 \mathrm{D}$ lengths $[6,25]$.

In this Letter we answer three critical questions about the DL first described in Ref. [6]; namely, we make simultaneous measurements of the plasma potential and ion beam, confirming both the presence of an ion beam and the existence of a strong electric structure, we demonstrate that the thickness of the DL is on the order of $20 \mathrm{D}$ lengths, and we show that the DL is tied to the magnetic field at a position close to the maximum in the gradient and half the maximum of the field. In addition, we have extended the study to lower pressures and have demonstrated that the DL scales up with source and diffusion chamber diameter.

The large volume helicon diffusion system WOMBAT (waves of magnetized beams and turbulence) is described extensively elsewhere [26], but briefly consists of a $20 \mathrm{~cm}$ diameter, $50 \mathrm{~cm}$ long Pyrex source tube connected con- 
tiguously and on axis to a $1 \mathrm{~m}$ diameter, $2 \mathrm{~m}$ long stainless steel diffusion chamber. At its other end, the source tube is terminated with an aluminum plate through which a moveable Pyrex plate attached to an axially translating vacuum feedthrough is inserted. The plate is roughly the inner diameter of the source and serves to alter its effective length while maintaining an insulated boundary for the plasma (the entire source was nonconducting). A steady axial magnetic field of up to $250 \mathrm{G}$ was provided by two external solenoids mounted concentrically with the source tube. The solenoids were mounted on sliding collets that allowed the axial position of the solenoids to be altered relative to the source but not to each other. Up to $600 \mathrm{~W}$ of rf power was provided at $7.2 \mathrm{MHz}$ to a coaxial double saddle antenna via a Pi matching circuit and was mounted $21 \mathrm{~cm}$ from the source end plate. The antenna was $15 \mathrm{~cm}$ long and was constructed from $3 \mathrm{~mm}$ thick $10 \mathrm{~mm}$ wide gold plated copper strap. Argon feed gas was supplied via a mass flow controller at the downstream end of the diffusion chamber.

A retarding field energy analyzer (RFEA) mounted on axis was employed to measure the ion energy distribution function (IEDF) and the plasma density as a function of axial position. Mounted in the diffusion chamber, the probe collection surface was always parallel to the exit plane of the source and allowed for the simultaneous measurements of the local plasma potential, the beam energy, and the beam current density as a function of axial position. The position, thickness, and potential drop of the double layer could therefore also be determined. The probe construction and analysis followed the design and methods described in detail by Charles et al. [27] and will not be presented again here.

A $5 \mathrm{~mm}$ long, $250 \mu \mathrm{m}$ diameter cylindrical Langmuir probe was used to measure the electron temperature $35 \mathrm{~cm}$ downstream from the source and to calibrate the RFEA plasma density measurements. The probe could translate radially and was used to measure $T_{e}$ on axis using a standard analysis. Using this probe it was shown that the floating potential, electron temperature, and local plasma potential were not affected by the presence of the RFEA. Measurements were taken for 5 axial positions of the RFEA: one upstream of the double layer, one just downstream of the double layer, and three further downstream in the diffusion chamber. With the Langmuir probe $2 \mathrm{~cm}$ off center (radially), the floating potential, plasma potential, and electron temperature for these measurements were $10 \mathrm{~V}, 38 \mathrm{~V}$, and $6 \mathrm{eV}$, respectively, for a pressure of 0.15 mTorr, rf power of $500 \mathrm{~W}$, and a magnetic field of $180 \mathrm{G}$.

As can be seen in Fig. 1, the diffusion chamber, which is a grounded stainless steel vessel, has an $11 \mathrm{~cm}$ long, $26 \mathrm{~cm}$ diameter throat that leads into the main diffusion chamber via a rapidly expanding curvilinear bell. The junction between the insulated source tube and the diffusion chamber throat forms a right angle and is marked as zero in Figs. 1 and 2, dividing the system into the source region

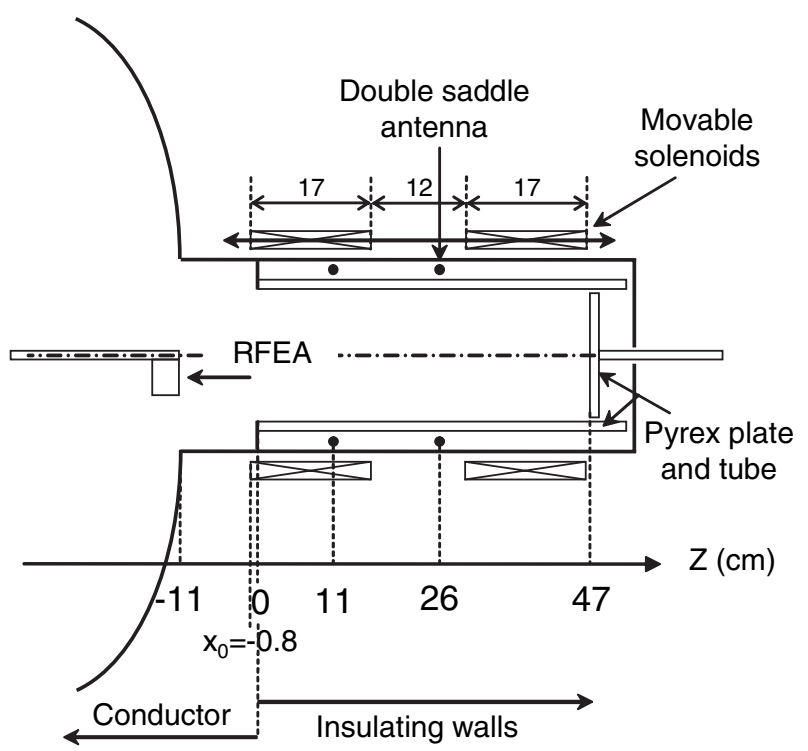

FIG. 1. Schematic of WOMBAT source.

(positive numbers) and the diffusion region (negative numbers). The bottom panel of Fig. 2 shows the local plasma potential on axis measured as a function of position for a pressure of $0.15 \mathrm{mTorr}$, rf power of $500 \mathrm{~W}$, and a magnetic field of $180 \mathrm{G}$. A discontinuity in the data can be seen $10.8 \mathrm{~cm}$ downstream of the source tube where the local plasma potential jumps from 35 to $58 \mathrm{~V}$ over a distance of no more than $5 \mathrm{~mm}$. This is the DL, downstream of which a clear beam can be seen in the ion energy distribution function as shown in Fig. 3. Upstream there is no beam and consequently the IEDF has only one peak corresponding to the upstream plasma potential. This appears to be quite constant as a function of position, although it was measured only over a distance of $5 \mathrm{~cm}$ due to a restriction in the axial movement of the probe. Between about 12 and $30 \mathrm{~cm}$ downstream of the DL, the potential drops off slowly at a rate of approximately $6.25 \mathrm{~V} \cdot \mathrm{m}^{-1}$. Beyond this the plasma potential is constant as a function of position and is $28 \mathrm{~V}$. The density as a function of axial position is shown in Fig. 4 and roughly follows the plasma potential. Based on the upstream density of $2.8 \times$ $10^{15} \mathrm{~m}^{-3}$ the thickness of the DL in D lengths is approximately 20.

The top panel of Fig. 2 shows the experimentally measured magnetic field (solid line) and the magnetic field gradient (dashed line) on axis as a function of position. The DL, represented by the dotted vertical line, is just downstream of the maximum in the magnetic field gradient or just upstream of half the maximum in the magnetic field. As the temperature of the solenoid windings was not taken into account during the measurement of the magnetic field or the DL, the location of the magnetic field relative to the two vertical lines can be given with only a certainty of $\pm 2 \mathrm{~mm}$.

The position of the DL relative to the magnetic field, however, was invariant, as shown in Fig. 5 where a clear 

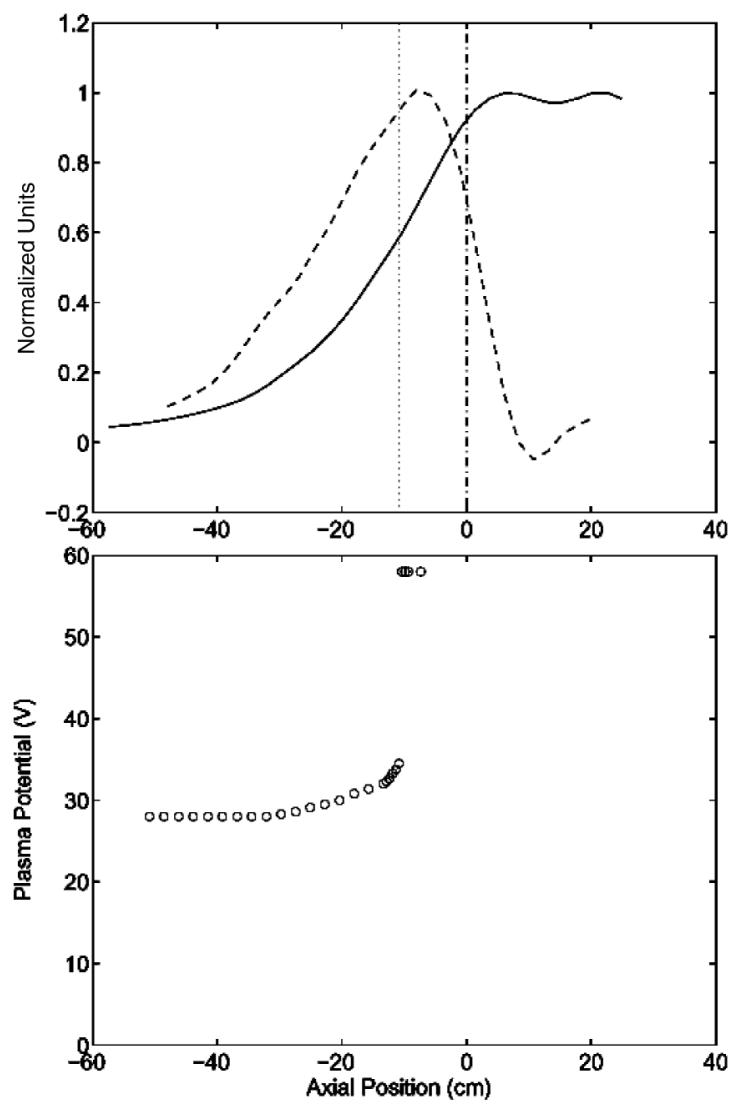

FIG. 2. Top panel: Magnetic field (solid line) and magnetic field gradient (dashed line) as a function of position; vertical lines show the position of the end of the source (dot-dashed line) and the DL (dotted line). Bottom panel: Plasma potential as a function of axial position for 0.15 mTorr, $500 \mathrm{~W}$, and $180 \mathrm{G}$ (=1 in normalized units).

linear relationship between the axial position of the DL and the axial position of the magnetic field can be discerned. The solenoids were translated together as a unit on sliding collets and their position was measured using their most downstream surface relative to the end of the source tube.

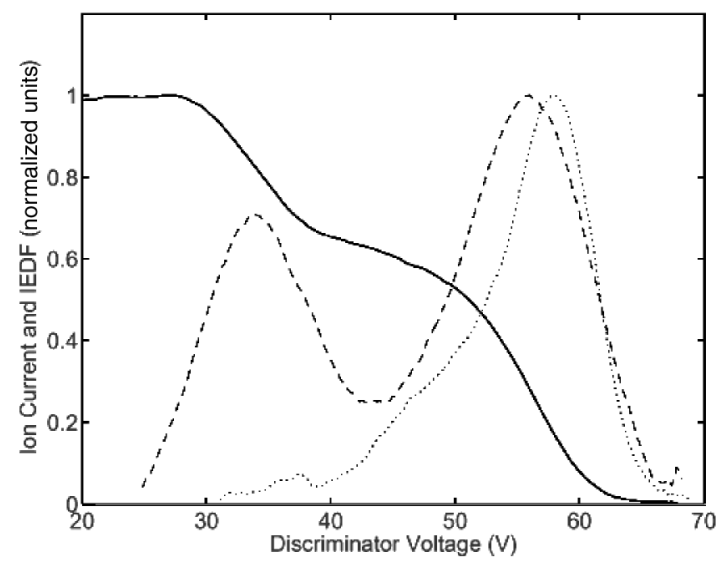

FIG. 3. Ion saturation current (solid line) and IEDF (dashed line) as measured $5 \mathrm{~cm}$ downstream of the DL for $0.15 \mathrm{mTorr}$, $500 \mathrm{~W}$, and $180 \mathrm{G}$. Upstream IEDF (dotted line).

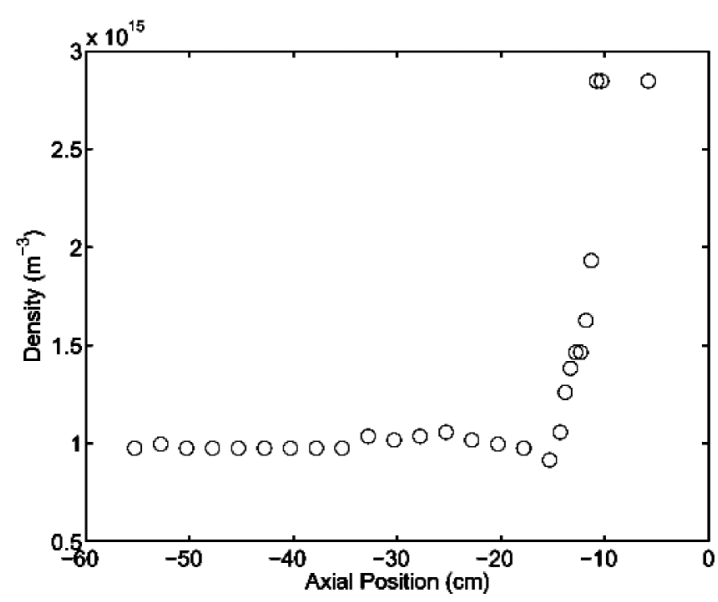

FIG. 4. Density as a function of axial position for $0.15 \mathrm{mTorr}$, $500 \mathrm{~W}$, and $180 \mathrm{G}$. DL at $-10.8 \mathrm{~cm}$.

Their upstream range of motion was restricted to $3 \mathrm{~cm}$ due to the position of the antenna leads and for positions further downstream than $1 \mathrm{~cm}$ from the end of the source tube no DL could be obtained. For these downstream points, both the maximum in the magnetic field gradient and the half maximum of the magnetic field were outside the throat of the diffusion chamber in the region where the chamber diameter expands rapidly. With the exception of Fig. 5, the data presented in this letter correspond to a solenoid position of $-0.8 \mathrm{~cm}$.

Figure 6 shows the evolution of the upstream plasma potential, the potential drop across the DL and the electron temperature as a function pressure. The pressure range is from 0.09 to $0.3 \mathrm{mTorr}$ and extends the data presented in Ref. [28]. Down to 0.15 mTorr, the potential drop across the DL fits the log-linear relationship of Fig. 4 in Ref. [28]. For lower pressures, however, the strength of the double layer rises sharply. It is noted that the electron temperature (measured downstream) was relatively low for the pressures employed, varying between $4.7 \mathrm{eV}$ at $0.3 \mathrm{mTorr}$ and $6.4 \mathrm{eV}$ at $0.1 \mathrm{mTorr}$ but correlated well with previous

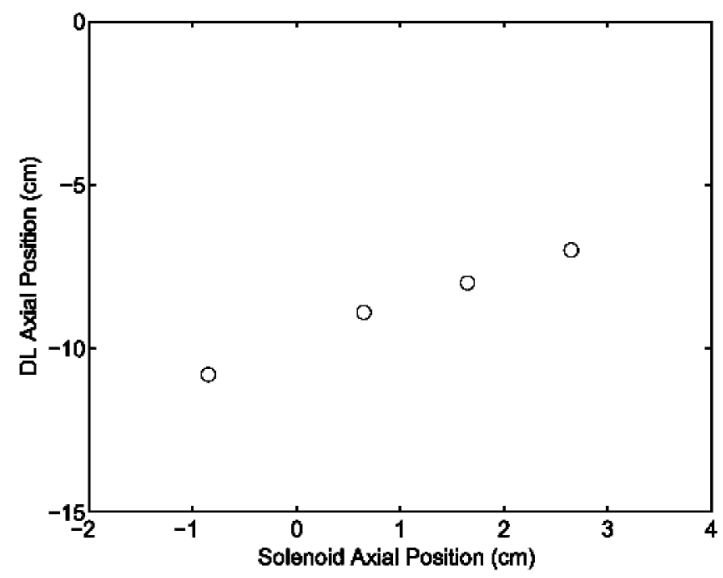

FIG. 5. Variation of the DL position relative to the end of the source as a function of the magnetic field position. 


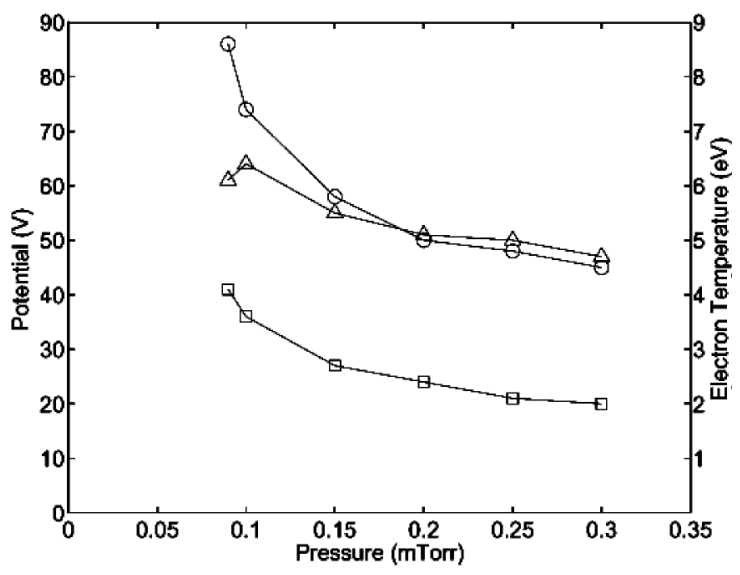

FIG. 6. (○) Plasma potential upstream of the DL; ( $\square$ ) potential drop across the DL; $(\triangle)$ electron temperature. Operating conditions: $0.15 \mathrm{mTorr}$ and $180 \mathrm{G}$.

measurements in WOMBAT. Moreover, the results are consistent with the inverse relationship between electron temperature and pressure, and the log of the electron current was linear over several decades. In addition, the electron temperature deduced from $\left(V_{p}-V_{f}\right) / 4.7$ correlated well with that measured from the slope of the electron current of the Langmuir probe.

As a consequence of this relatively low electron temperature, the strength of the $\mathrm{DL}\left(\phi_{\mathrm{DL}} / T_{e}\right)$ in WOMBAT was approximately 5 , which was somewhat higher than the value of 3 previously found by Ref. [6]. Below 0.15 mTorr, this figure rose sharply attaining 7 at 0.09 mTorr.

In summary, by simultaneous measurements of the axial potential and ion distribution function, we have shown that the current-free DL created at low pressure in an expanding plasma clearly accelerated ions and that it has a thickness of less than $20 \mathrm{D}$ lengths rather than the $50 \mathrm{D}$ lengths published earlier [6]. Perhaps more importantly, we have demonstrated that the DL tracks with the moving magnetic field structure, indicating that it is created independently of the plasma source or of the physically expanding geometry of the experiment. This lends confidence to its application in extraterrestrial situations.

N.P. wishes to thank the SP3 group of the Plasma Research Laboratory at ANU for their hospitality during his six-weeks stay, and Dr. P. Chabert and Professor R. W. Boswell for providing the funding.
[1] N. Singh and R. W. Schunk, J. Geophys. Res. 87, 9154 (1982).

[2] M. A. Raadu, Phys. Rep. 178, 25 (1989).

[3] M. Temerin, M.H. Boehm, and F.S. Mozer, Geophys. Res. Lett. 8, 799 (1981).

[4] M. Temerin, K. Cerny, W. Lotko, and F. S. Mozer, Phys. Rev. Lett. 48, 1175 (1982).

[5] R. E. Ergun et al., Phys. Rev. Lett. 87, 045003 (2001).

[6] C. Charles and R. Boswell, Appl. Phys. Lett. 82, 1356 (2003).

[7] C. Chan and N. Hershkowitz, Phys. Fluids 25, 2135 (1982).

[8] S. Torven, Phys. Rev. Lett. 47, 1053 (1981).

[9] N. Sato et al., Phys. Rev. Lett. 46, 1330 (1981).

[10] M. Guyot and C. Hollenstein, Phys. Fluids 26, 1596 (1983).

[11] S. Iizuka, K. Saeki, N. Sato, and Y. Hatta, Phys. Rev. Lett. 43, 1404 (1979).

[12] G. Knorr and C. K. Goertz, Astrophys. Space Sci. 31, 209 (1974).

[13] G. Joyce and R.F. Hubbard, J. Plasma Phys. 20, 391 (1978).

[14] M. K. Hudson, W. Lottko, I. Roth, and E. Witt, J. Geophys. Res. 88, 916 (1983).

[15] P. Coakley and N. Hershkowitz, Phys. Fluids 22, 1171 (1979).

[16] C. Hollenstein, M. Guyot, and E. S. Weibel, Phys. Rev. Lett. 45, 2110 (1980).

[17] S. Iizuka et al., Phys. Rev. Lett. 48, 145 (1982).

[18] C. Chan, N. Hershkowitz, and G. L. Payne, Phys. Lett. 83A, 328 (1981).

[19] G. Hairapetian and R. L. Stenzel, Phys. Rev. Lett. 61, 1607 (1988).

[20] N. Plihon, C. S. Corr, and P. Chabert, Appl. Phys. Lett. 86, 091501 (2005).

[21] R. Plamondon, J. Teichmann, and S. Torven, J. Phys. D 21, 286 (1988).

[22] B. Song, R. L. Merino, and N. D’Angelo, Phys. Scr. 45, 395 (1992).

[23] X. Sun, C. Biloiu, R. Hardin, and E. E. Scime, Plasma Sources Sci. Technol. 13, 359 (2004).

[24] S. A. Cohen et al., Phys. Plasmas 10, 2593 (2003).

[25] C. Charles and R. W. Boswell, Phys. Plasmas 11, 1706 (2004).

[26] R. W. Boswell and R. K. Porteous, Appl. Phys. Lett. 50, 1130 (1987).

[27] C. Charles, A. W. Degeling, T. E. Sheridan, J. H. Harris, M. A. Lieberman, and Boswell, Phys. Plasmas 7, 5232 (2000).

[28] C. Charles, Phys. Plasmas 12, 044508 (2005). 